5. Quant aux laits secs, - lorsqu'on envisage leur pouvoir antiscorbutique une fois reconstituée - , ils se rapprochent des laits ayant subi une stérilisation par autoclavage. Leur teneur en vitamine $\mathrm{C}$ est loin d'être négligeable, surtout pour certains d'entre eux (voir le tableau VI).

Nous insisterons une fois de plus, pour terminer, sur la nécessité de donner aux enfants consommant des laits de vache bouillis, stérilisés, concentrés ou secs, un supplément quotidien de jus de fruits riches en vitamine C, de jus d'orange, par exemple.

Lorsqu'on dirige l'alimentation de nourrissons soumis à l'allaitement artificiel, il est bon d'avoir présent à l'esprit ce fait- que le lait d'une mère bien portante apporte, à son enfant nourri au sein, de $40 \mathrm{mgr}$. à $45 \mathrm{mgr}$. de vitamine $C$ par litre, tandis que le lait frais d'une vache n'en apporte que 15 à 25 milligrammes, selon la saison et selon le mode d'alimentation (1).

(Travail du Laboratoire de Physiologie de la Nutrition de l'Ecole des Hautes-Etudes et du Centre de Recherches scientifiques sur l'alimentation de l'Institut National de la Recherche agronomique.)

\title{
NOTE SUR LE DOSAGE DES CHLORURES DANS LES LAITS (2)
}

par

\section{DURON et MHe A. FOURNIER}

Des expériences que nous avons effectuées sur les laits du Service, il résulte que la méthode publiée par MM. Massot et Lestra (Annales des Falsifications, 1935, p. 608) est la plus intéressante. En effet, elle présente les avantages de donner en milieu alcoolique un virage net du sulfocyanure et de réduire rapidement le bichromate en sel de chrome dont la faible coloration verte ne gêne pas ou facilite même l'apparition de la coloration rouge du sulfocyanure ferrique.

Le seul reproche que nous puissions faire à cette méthode, comme à toute autre analogue, est d'employer une défécation au $1 / 10^{\circ}$. Dans ces conditions, la prise d'essai du filtrat étant de $75 \mathrm{~cm}^{3}$, le coefficient de calcul est de 0,78 , ce qui donne, la lecture de la burette s'effectuant à $0 \mathrm{~cm}^{3} 05$ près, une erreur minima d'environ $4 \mathrm{cgr}$. de $\mathrm{NaCl}$ par litre, soit après calcul, environ $0 \mathrm{~cm}^{3} 5$.

(1) Voir Tables de composition des Aliments, par L. Randoin, P. Le Gallic J. Causeret, 1947. Lanore, éditeur. (An. in Le Lait, 1947, 268, 481.)

(2) Ann. Fals. Fr., 1948, 478-480, 537. 
Nous avons fait les constatations suivantes:

1. L'acétone n'est pas indispensable à la défécation. Celle-ci peut se faire avec un alcool pur à $95^{\circ}$ ou avec un alcool dénaturé exempt d'ion $\mathrm{Cl}$.

2. La défécation s'effectue aussi bien au $1 / 5^{\mathrm{e}}$ qu'au $1 / 10^{\mathrm{e}}$.

C'est pour ces raisons que nous opérons selon la méthode Massot-Lestra, mais en employant $20 \mathrm{~cm}^{3}$ de lait. Le calcul est alors :

$$
\text { (5-n) } 0,00585 \frac{1.000}{20} \cdot \frac{4}{3}=(5-n) 0,39 \mathrm{NaCl} \text { par litre. }
$$

ce qui donne une erreur minima de $2 \mathrm{cgr}$. par litre.

Dans ces conditions, il est possible de doser jusqu'à 1 gr. 90 de $\mathrm{NaCl}$ par litre. Si la teneur est supérieure, il suffit de rajouter $5 \mathrm{~cm}^{3}$ de $\mathrm{NO}_{3} \mathrm{Ag} \mathrm{N} / 10$ et d'effectuer le calcul sur (10-n).

\section{RECHERCHES EXPÉRIMENTALES SUR LE LAIT DE. VACHE ACTINISÉ \\ III. INFLUENCE DE L'ACTINISATION SUR LA TENEUR DU LAIT EN VITAMINE A (1) \\ par}

\section{JEANNE BOISSELOT et JEAN CAUSERET}

Bien que la, vitamine A soit sensible aux radiations ultraviolettes, il ne semble pas que l'irradiation des produits laitiers, lorsqu'elle est pratiquée en vue d'augmenter leur pouvoir antirachitique, diminue d'une manière notable leur teneur en cette vitamine.

Dès 1933, Bender et Supplee [1] ont constaté que, si la diminution de l'activité vitaminique A du lait au cours de son irradiation dépend de la technique employée, il n'en est pas moins possible d'exposer cet aliment aux rayons ultra-violets pendant un temps assez prolongé (jusqu'à 48 secondes) sans affecter vraiment sa teneur en vitamine $A$. Ces faits ont été confirmés au cours des années suivantes par Schoenleber, StemnBock et BAUMAN [2], puis par Callon et Hixon [3]. Les premiers auteurs montrèrent que l'irradiation du lait ordinaire et du lait concentré ne modifie pas leur teneur en vitamine A et en carotène; les seconds obtinrent des courbés de croissance identiques au moyen de rats qui recevaient,

(I) Bull, Soc. Sc, Hyg. alim., 1948, 36, 30. 